\title{
The muscle-specific ubiquitin ligase atrogin-1/MAFbx mediates statin-induced muscle toxicity
}

\author{
Jun-ichi Hanai, ${ }^{1}$ Peirang Cao, ${ }^{1}$ Preeti Tanksale, ${ }^{1}$ Shintaro Imamura, ${ }^{2,3}$ \\ Eriko Koshimizu, ${ }^{3,4}$ Jinghui Zhao, ${ }^{5}$ Shuji Kishi, ${ }^{3}$ Michiaki Yamashita, ${ }^{2}$ \\ Paul S. Phillips, ${ }^{6}$ Vikas P. Sukhatme, ${ }^{1}$ and Stewart H. Lecker ${ }^{1}$
}

\begin{abstract}
${ }^{1}$ Renal Division and Division of Interdisciplinary Medicine and Biotechnology, Department of Medicine, Beth Israel Deaconess Medical Center, Boston, Massachusetts, USA. ${ }^{2}$ National Research Institute of Fisheries Science, Yokohama, Japan. ${ }^{3}$ Schepens Eye Research Institute, Harvard Medical School, Boston, Massachusetts, USA. ${ }^{4}$ Tokyo University of Marine Science and Technology, Tokyo, Japan. ${ }^{5}$ Department of Cell Biology, Harvard Medical School, Boston, Massachusetts, USA. ${ }^{6}$ Scripps Mercy Hospital, San Diego, California, USA.
\end{abstract}

\begin{abstract}
Statins inhibit HMG-CoA reductase, a key enzyme in cholesterol synthesis, and are widely used to treat hypercholesterolemia. These drugs can lead to a number of side effects in muscle, including muscle fiber breakdown; however, the mechanisms of muscle injury by statins are poorly understood. We report that lovastatin induced the expression of atrogin-1, a key gene involved in skeletal muscle atrophy, in humans with statin myopathy, in zebrafish embryos, and in vitro in murine skeletal muscle cells. In cultured mouse myotubes, atrogin-1 induction following lovastatin treatment was accompanied by distinct morphological changes, largely absent in atrogin-1 null cells. In zebrafish embryos, lovastatin promoted muscle fiber damage, an effect that was closely mimicked by knockdown of zebrafish HMG-CoA reductase. Moreover, atrogin-1 knockdown in zebrafish embryos prevented lovastatin-induced muscle injury. Finally, overexpression of PGC-1 $\alpha$, a transcriptional coactivator that induces mitochondrial biogenesis and protects against the development of muscle atrophy, dramatically prevented lovastatin-induced muscle damage and abrogated atrogin-1 induction both in fish and in cultured mouse myotubes. Collectively, our human, animal, and in vitro findings shed light on the molecular mechanism of statin-induced myopathy and suggest that atrogin-1 may be a critical mediator of the muscle damage induced by statins.
\end{abstract}

\section{Introduction}

Statins (HMG-CoA reductase inhibitors) are among the most commonly prescribed medications in developed countries, with almost 500,000,000 prescriptions dispensed as of 2001 (1). They impair cholesterol production by inhibiting the synthesis of mevalonate, the rate-limiting step in the cholesterol biosynthetic pathway. Statins are generally well tolerated but can produce a variety of skeletal muscle-associated, dose-dependent adverse reactions, ranging from muscle pain to muscle cell damage and severe rhabdomyolysis (2). The frequency of rhabdomyolysis is low, with a reported incidence of approximately 1 per 10,000; however, fatalities have been reported $(1,3,4)$. Symptomatic muscle weakness and pain are much more frequent but difficult to quantitate, since objective measures of muscle damage such as elevation of creatine kinase in the serum of patients are usually absent $(5,6)$. Unfortunately, fear of muscle toxicity remains a major impediment preventing patients and their physicians from complying with statin therapy guidelines, and less than half of patients with indications for statins currently receive this therapy $(7,8)$.

Little is known about the molecular mechanisms by which HMG-CoA reductase inhibitors produce skeletal muscle injury $(2,9,10)$. Since mevalonate is an important precursor not only of cholesterol but also of ubiquinone (coenzyme $\mathrm{Q}_{10}\left[\mathrm{CoQ}_{10}\right]$ ), dolichols, and other

Nonstandard abbreviations used: $\mathrm{CoQ}_{10}$, coenzyme $\mathrm{Q}_{10}$ (ubiquinone); hpf, hour(s) post fertilization; MO, Morpholino antisense oligonucleotide.

Conflict of interest: The authors have declared that no conflict of interest exists. Citation for this article: J. Clin. Invest. 117:3940-3951 (2007). doi:10.1172/JCI32741. isoprenoids (11), muscle toxicity could be mediated by many different intracellular pathways. Indeed, inhibition of squalene synthase and squalene epoxidase, distal enzymes specific to only cholesterol biosynthesis, does not cause toxicity in cultured muscle cells $(12,13)$. Furthermore, recent experiments suggest that statins affect mitochondrial function (14). Interestingly, $\mathrm{CoQ}_{10}$, a component of the inner mitochondrial membrane required for oxidative phosphorylation, is prenylated; thus, its synthesis is inhibited by statins (15-17).

Skeletal muscle mass is determined by the competing processes of protein synthesis and protein breakdown. Rapid loss of muscle mass occurs in response to food deprivation and in many major disease states (e.g., cancer cachexia, diabetes, uremia, cardiac failure, sepsis) and also with disuse $(18,19)$. In these conditions, muscle protein is rapidly mobilized through a common cellular mechanism involving similar biochemical and transcriptional adaptations, including activation of the ubiquitin proteasome pathway (UPP), the main intracellular system for protein degradation $(20,21)$. Among the UPP components induced in atrophying muscle is the ubiquitinprotein ligase or $\mathrm{E} 3$, atrogin-1/MAFbx $(22,23)$. Atrogin-1 is induced early during the atrophy process, and the rise in atrogin- 1 expression precedes the loss of muscle weight (22). Animals lacking atrogin1 are resistant to muscle atrophy following denervation (23), suggesting that atrogin-1 targets key muscle protein(s) for destruction, though the identity of these component(s) is still unclear. Recent studies have demonstrated induction of atrogin-1 in other dying or involuting tissues. For example, it is induced in cardiac muscle in failing hearts $(24,25)$, in uterine smooth muscle in the postpartum period as the uterus involutes to its resting size (26), and in dying 


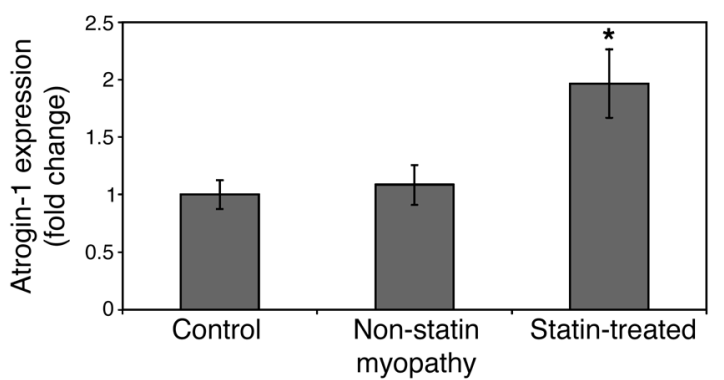

Figure 1

Atrogin-1 is induced in human biopsy samples from patients with statin-induced muscle injury. Total RNA was extracted from human quadriceps muscle biopsies, and atrogin-1 mRNA was quantitated by real-time PCR as described in Methods. ${ }^{*} P=0.017$, difference between groups by 1 -way ANOVA.

stromal tumors responding to imatinib treatment (27). We reasoned that since atrogin-1 plays a key role in activating protein breakdown in a wide range of tissues, especially skeletal muscle, it might also mediate part of their sensitivity to statins.

Distinct muscle beds have different susceptibilities to protein loss and atrophy. In rodents, type II, intermittently used, pale muscle fibers are atrophy prone while type I dark muscles are relatively resistant (28-31). These dark fibers/muscles have a high volume of mitochondria, high capillary density, and high oxidative enzyme capacity $(32,33)$ while the pale fibers/muscles are largely glycolytic. It is likely that the decreased mitochondrial number/function in pale, glycolytic muscle enhances susceptibility to muscle damage and wasting. The PGC-1 family of transcriptional coactivators has been recently discovered to activate a transcriptional program of mitochondrial biogenesis and oxidative metabolism and is critical in the maintenance of glucose, lipid, and energy homeostasis in muscle and other tissues $(34,35)$. In skeletal muscle, PPAR $\gamma$ coactivator $1 \alpha$ (PGC- $1 \alpha)$ functions as a critical metabolic sensor of calcium-induced contractile activity (36-39). Its expression is induced by both short-term and chronic exercise in rodents and humans $(39,40)$. Transgenic expression of PGC-1 $\alpha$ in fast-twitch, glycolytic muscles transforms the type IIb muscle fibers into a more oxidative phenotype (41). PGC-1 $\alpha$ therefore appears to be an important mediator in regulating mitochondrial metabolic properties in skeletal muscle and is a prime candidate for a regulator that might protect against muscle damage and atrophy through its ability to augment mitochondrial number and function. Indeed, rodent muscles show a large decrease in PGC-1 $\alpha$ mRNA during atrophy induced by denervation as well as by cancer cachexia, diabetes, and renal failure (21). Furthermore, in transgenic mice overexpressing PGC- $1 \alpha$, denervation and fasting caused a much smaller decrease in muscle fiber diameter and a smaller induction of atrogin-1 than in control mice (42).

In the present study, we demonstrate that statins induce marked induction of atrogin-1 expression in human skeletal muscle, cultured muscle cells, and an animal model of statin myopathy in zebrafish. Furthermore, in the absence of atrogin-1, cells and animals are resistant to the toxic effects of statins. Forced overexpression of PGC-1 $\alpha$ suppresses statin-induced atrogin-1 expression and protects from statin-induced muscle damage. We conclude that atrogin-1 mediates part of statin's effects in muscle and that inhibition of atrogin-1 function might protect against the detrimental effects of these agents.

\section{Results}

Statin toxicity in human muscle biopsies is associated with atrogin-1 expression. Since the muscle-specific ubiquitin protein ligase atrogin-1 is commonly induced in diverse states of muscle wasting $(20,21)$, we examined its expression in statin-treated patients with symptoms of muscle pain or weakness. We measured by real-time PCR atrogin-1 levels in 19 human quadriceps muscle biopsies from 5 patients undergoing knee replacements (controls), from 6 patients with muscle pain but not being treated with HMG-CoA reductase inhibitors, and from 8 patients with muscle pain/damage concomitantly being treated with these medications (Supplemental Table 1; supplemental material available online with this article; doi:10.1172/JCI32741DS1). Interestingly, atrogin-1 expression was significantly higher in the statin-treated muscle samples (Figure 1). Though the subjects were not strictly sex-matched, we observed generally higher atrogin-1 mRNA levels in both males and females who had been administered HMG-CoA reductase inhibitors, suggesting that the difference was not sex-related (Supplemental Figure 1). These human data point to a potential role for atrogin-1 in the muscle toxicity of statin medications.

Lovastatin causes atrogin-1 induction in cultured myotubes. To begin to study the effects of HMG-CoA reductase inhibitors on muscle cells, we treated differentiated $\mathrm{C} 2 \mathrm{C} 12$ myotubes with various concentrations of the HMG-CoA reductase inhibitor lovastatin. Compared with control myotubes treated with an equal volume of vehicle, in the presence of increasing concentrations of lovastatin, myotubes became progressively thinner and appeared to have more cytoplasmic vacuolation, changes in cell contour, and frank disruption or loss of myotubes (Figure 2). The reduction in myotube size was quantitated by measurement of myotube thickness. This effect was clearly visible at low lovastatin concentrations, in the range of those typically found in patients administered this medication $(43,44)$. Morphological changes were visible in the myotube cultures after 24 hours of treatment, with almost complete loss of myotubes by 5 days (Figure 2B). These effects were not unique to lovastatin, as similar results were seen when cultures were treated with another HMG-CoA reductase inhibitor, cerivastatin (data not shown).

Since lovastatin caused a reduction in myotube size and integrity, we next measured whether it led to induction of genes activated in muscle undergoing atrophy. Since atrogin-1 is the most highly induced gene in atrophying muscle from diverse causes $(20,21)$, we measured its expression in these lovastatin-treated myotube cultures. Using real-time PCR, we found that atrogin-1 mRNA was dramatically and rapidly induced by lovastatin in a time- and concentration-dependent manner (Figure 3A). At the highest lovastatin concentration $(10 \mu \mathrm{M})$, atrogin-1 mRNA was significantly increased at 6 hours and induced as much as 6 -fold by 36 hours of treatment. We also assessed atrogin-1 protein levels and found that the mRNA increases were mirrored by increases in protein. At low lovastatin concentration $(1.0 \mu \mathrm{M})$ for 48 hours, atrogin-1 induction was about 1.5 -fold, and at high concentration $(10 \mu \mathrm{M})$, it increased about 2.5 -fold compared with nontreated control cells. This amount of atrogin-1 activation was similar to that found in myotubes atrophying due to dexamethasone treatment (ref. 45; Figure 3B). We next measured rates of protein breakdown in these lovastatin-treated myotube cultures since induction of atrogin-1 has been correlated with enhanced proteolysis (46). As with dexamethasone, a known inducer of atrogin-1, proteolytic rate, and muscle atrophy (45-47), lovastatin led to a consistent $5-10 \%$ increase in the rate of bulk muscle proteolysis compared with con- 
A
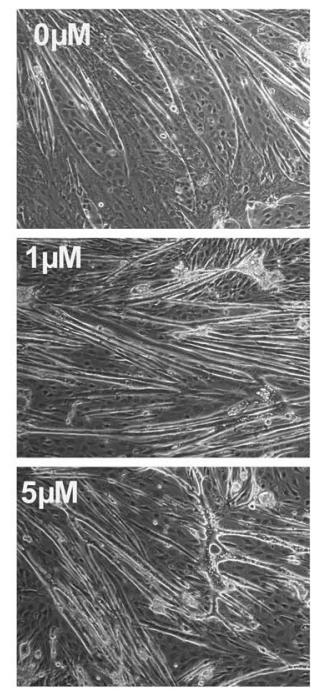

B
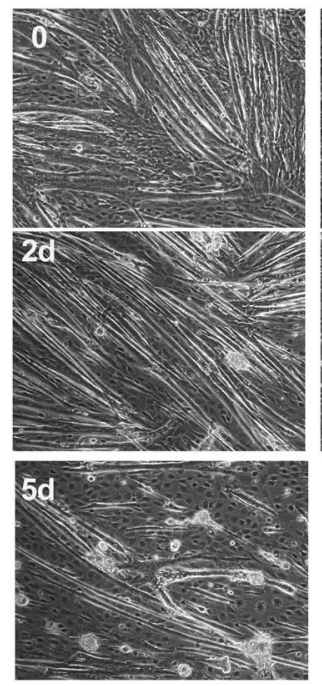

Figure 2

Lovastatin causes reduction in myotube diameter. C2C12 myotube morphology and mean diameter following treatment with lovastatin at various concentrations $(\mathbf{A})$ or for various times $(\mathbf{B})$. Control $(0 \mu \mathrm{M})$ cultures were treated with reagent vehicle alone. Original magnification, $\times 100$.
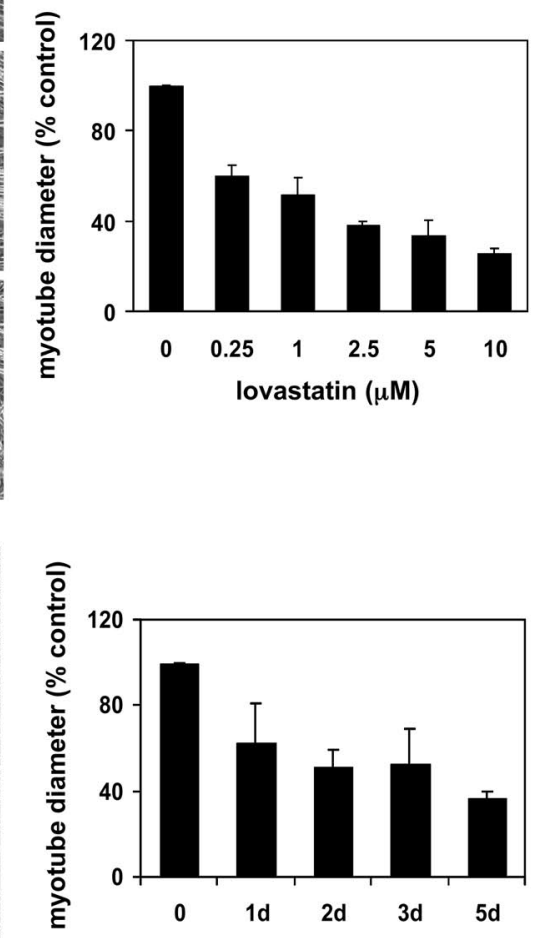
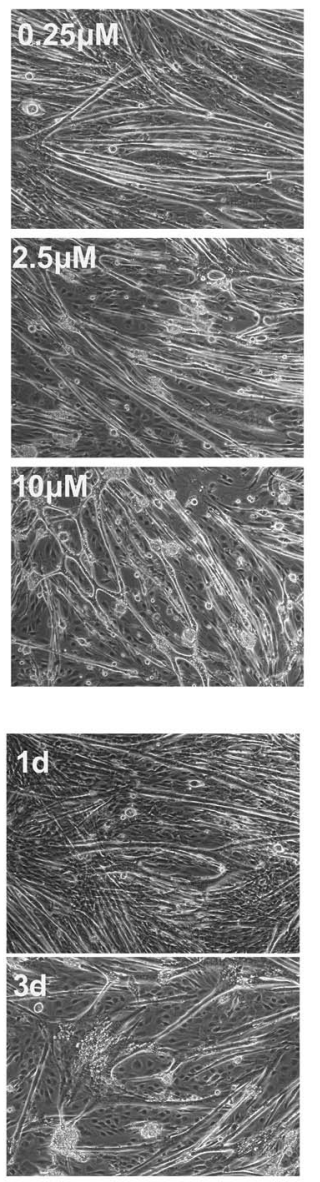

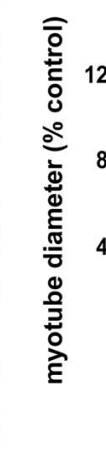

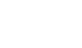

changes in the atrogin-1-containing primary myotubes compared with C2C12 cells. Interestingly, primary myotubes lacking atrogin-1 had less damage than control cells at similar lovastatin concentrations (Figure 4B). In the atrogin-1 null cells treated with 0.25 and $1.0 \mu \mathrm{M}$ lovastatin, little change in myotube size was noted whereas in the control cultures, tube diameter decreased by as much as $50 \%$ after 2 days of exposure to drug. These results clearly demonstrate that atrogin-1 is an important factor in lovastatin-induced myotube damage.

Lovastatin promotes damage of muscle fibers in zebrafish embryos. We next sought to develop an in vivo model to further study effects on muscle of lovastatin administration. Since wholebody muscle fibers can be stained in zebrafish embryos at 48 hours post fertilization (hpf) (48) and zebrafish are amenable to rapid genetic manipulations, we subjected zebrafish embryos to lovastatin. Embryos were treated with lovastatin from $20 \mathrm{hpf}$ to $32 \mathrm{hpf}$ at different concentrations $(0-5 \mu \mathrm{M})$. As in mammalian muscle cell culture, lovastatin led to clear dose-dependent muscle phenotypes, demonstrated by longitudinal muscle fiber staining with an antibody to myosin heavy chain (Figure 5). Muscle damage at low lovastatin concentration $(0.025-0.05 \mu \mathrm{M})$ was evidenced by bowing, gap formation, and fiber disruption (class 1 changes). At higher lovastatin concentration $(0.05-0.5 \mu \mathrm{M})$, fiber damage was more severe. Fiber thinning and attenuation of staining with the MHC antibody was frequently seen (class 2 changes). At maximal lovastatin concentration $(1.0-5.0 \mu \mathrm{M})$,

trol cultures (Figure 3C). These experiments suggest that statininduced muscle injury mediated by atrogin- 1 shares mechanistic features with atrophying muscle in which rates of muscle protein breakdown are generally increased.

To determine whether atrogin-1 expression is necessary for lovastatin-mediated damage, we made use of primary myotubes generated from mice lacking atrogin-1 (23). Since atrogin-1 null mice are resistant to muscle atrophy (23), we hypothesized that myotubes lacking atrogin-1 might also be resistant to lovastatin-induced damage. Primary myotubes derived from these mice were morphologically identical to cells from atrogin-1 wild-type control littermates (Figure 4B). These myotubes indeed contained no atrogin-1 protein, and the control primary myotubes activated atrogin- 1 expression after dexamethasone treatment or FoxO adenoviral infection (45) in a similar manner to the immortalized C2C12 myotubes (Figure 4A). Lovastatin treatment caused very similar morphological damage beyond the muscle was observed, with the development of irregular somite boundaries (class 3 changes). Using this classification, we found that class 3 changes were observed in over $60 \%$ of embryos subjected to $5 \mu \mathrm{M}$ lovastatin; however, more than $50 \%$ of embryos treated with concentrations 10 -fold lower (i.e., $0.05 \mu \mathrm{M}$ ) still demonstrated milder, class 1 defects (Figure 5B).

To confirm that lovastatin's effect on zebrafish muscle was due to the inhibition of HMG-CoA reductase rather than to another off-target effect, we knocked down the zebrafish HMG-CoA reductase gene ( $z-H M G$-CoA reductase) in zebrafish embryos using both missense and antisense morpholino oligonucleotides targeting the ATG region of the gene (ATG morpholino). Depletion of $z-H M G-$ CoA reductase showed similar effects as lovastatin treatment in zebrafish muscle fibers (Figure 6). As further documentation of the role of $z$-HMG-CoA reductase in maintaining zebrafish muscle fiber morphology, we also depleted active $z-H M G-C o A$ reductase by 

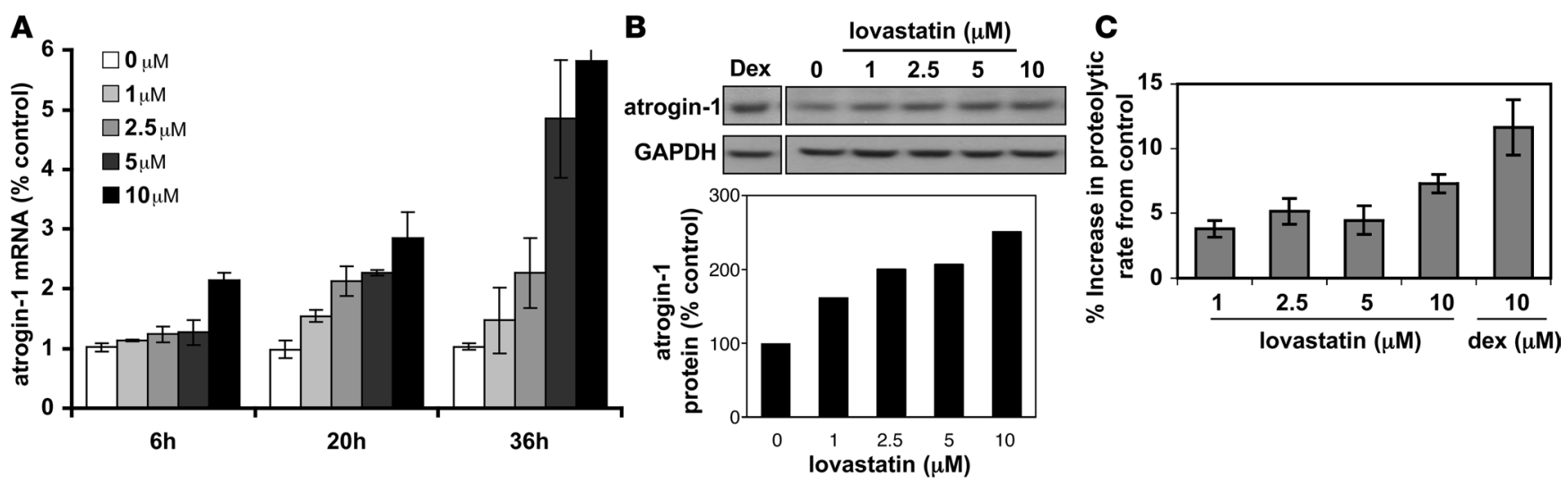

Figure 3

Lovastatin induces expression of both atrogin-1 mRNA and protein in cultured myotubes. (A) Atrogin-1 mRNA expression was measured by real-time PCR in samples of total RNA extracted from C2C12 myotubes treated with $0,1,2.5,5.0$, and $10 \mu \mathrm{M}$ lovastatin for 6 hours, 20 hours, and 36 hours, respectively. (B) C2C12 myotubes were treated with lovastatin for 48 hours at the indicated concentrations, protein lysates were prepared, and immunodetection using polyclonal anti-atrogin-1 antibody was performed as described in Methods. Atrogin-1 band intensity was quantitated by densitometry. Atrogin-1 expression induced by dexamethasone (5 $\mu \mathrm{M})(45)$ was used as a positive control. (C) Protein degradation was measured as described in Methods. Rates are presented as the percentage increase from proteolytic rate in nontreated control cultures. Dexamethasone $(10 \mu \mathrm{M})$ was used as a positive control.

creating a splicing morpholino oligonucleotide against the common splice site of both splice variants of the HMG-CoA reductase gene in zebrafish (Supplemental Figure 2). This mutant showed an abnormal muscle fiber structure similar to the $\mathrm{z}-\mathrm{HMG}-\mathrm{CoA}$ reductase knockdown (ATG morpholino) and wild-type embryos treated with lovastatin (Supplemental Figure 2).

Atrogin-1 knockdown prevents statin-induced and HMG-CoA reductase knockdown-induced muscle injury in zebrafish embryos. Since atrogin-1 is strongly induced in mammalian muscle cultures following lovastatin administration, we measured to determine whether it is also induced in lovastatin-treated zebrafish. Mouse and zebrafish atrogin- 1 are $75 \%$ identical and $86 \%$ similar at the amino acid level, and polyclonal antibodies to the mammalian protein recognize the zebrafish form (z-atrogin-1). As in mammalian cells, the zebrafish homolog of atrogin-1 was clearly and dose dependently elevated upon lovastatin treatment in the fish at both the mRNA (Figure 7A) and protein levels (Figure 7B). To determine whether atrogin-1 is required for the morphological effects of lovastatin on zebrafish muscle, we produced an antisense morpholino oligonucleotide against the atrogin-1 gene. Injection of this morpholino effectively knocked down endogenous atrogin-1 expression in zebrafish embryos (Figure 7C). No significant gross or histological abnormalities were observed in $\mathrm{z}$-atrogin-1-depleted embryos (Figure 7D). Wildtype embryos and $\mathrm{z}$-atrogin-1-depleted embryos were then treated with lovastatin $(0-1.0 \mu \mathrm{M})$. We detected significant rescue of the muscle damage phenotype in the $\mathrm{z}$-atrogin-1-depleted embryos

\section{Figure 4}

Myotubes from atrogin-1 null (-/-) mice are resistant to lovastatin-induced damage. (A) Atrogin-1 protein expression is absent in atrogin-1 null (-/-) myotubes. Myoblasts derived from atrogin-1 knockout mice $(-/-)$ and corresponding wild type $(+/+)$ littermates were differentiated into myotubes. Cultures were stimulated to express atrogin-1 with dexamethasone $(5 \mu \mathrm{M})$ or infected with constitutively active FoxO3- or GFP-expressing adenovirus (45). Atrogin-1 expression was detected by Western blotting as in Figure 3. (B) Myotubes from atrogin-1 null (-/-) and wild-type $(+/+)$ mice were treated with lovastatin at the indicated concentrations for 48 hours. Myotube morphology was examined, and diameter was measured. Original magnification, $\times 100$.
A

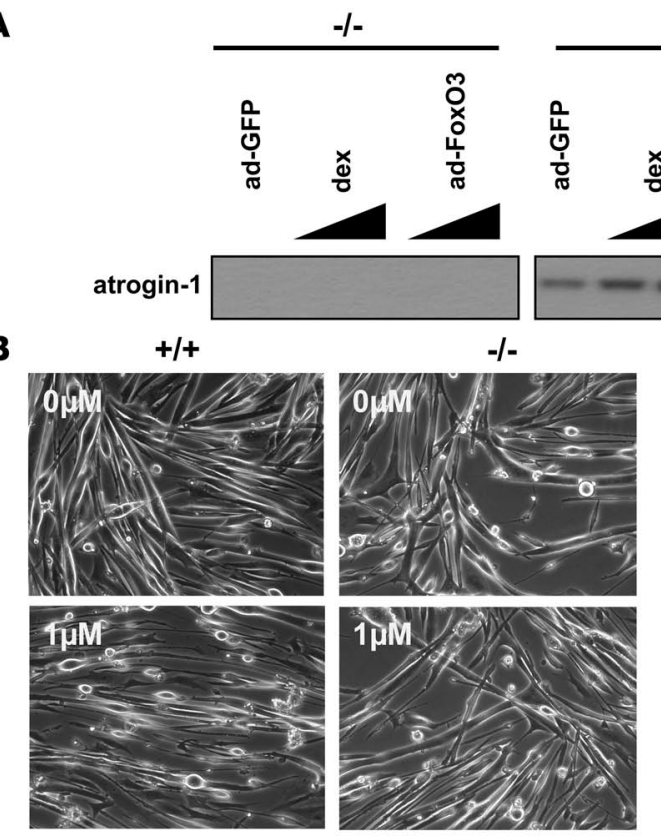

$+/+$
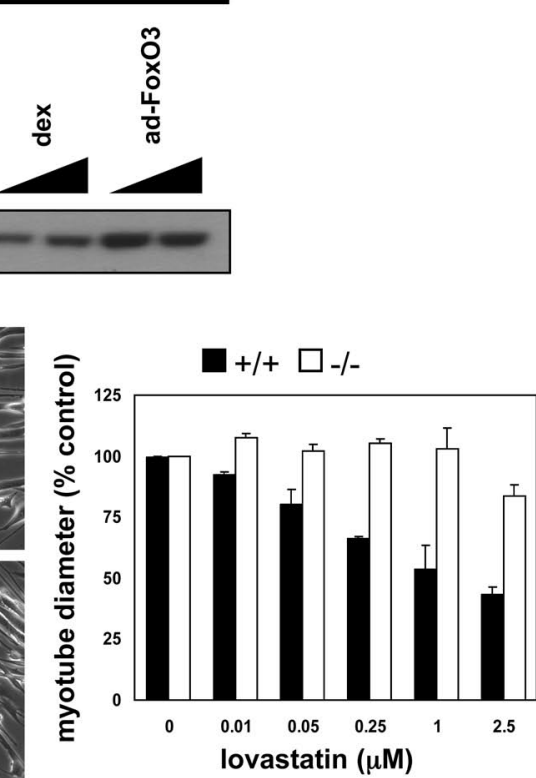
A

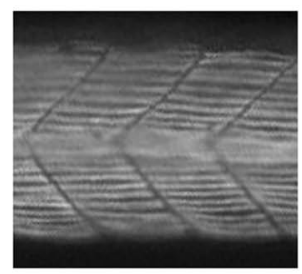

normal

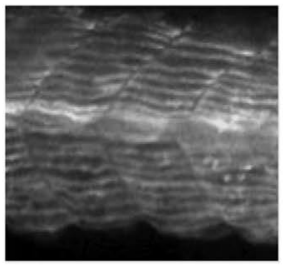

bowing

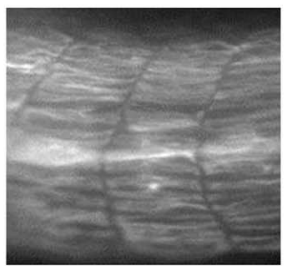

thin \&

irregular

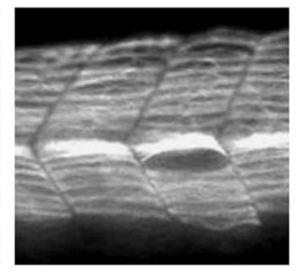

gap formation

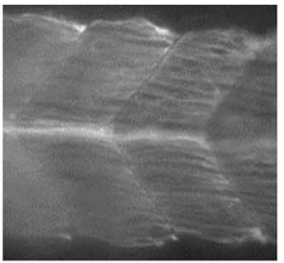

diffuse

appearance
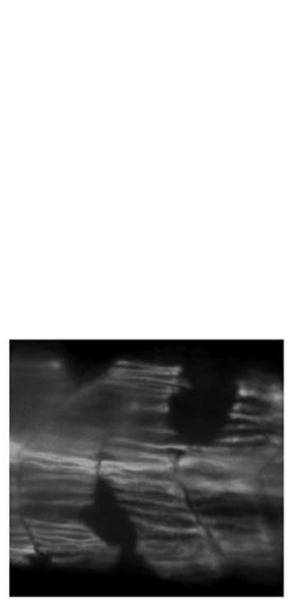

blocked \& disrupted

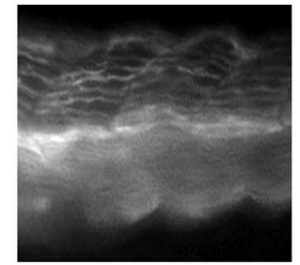

irregular somite boundaries
B

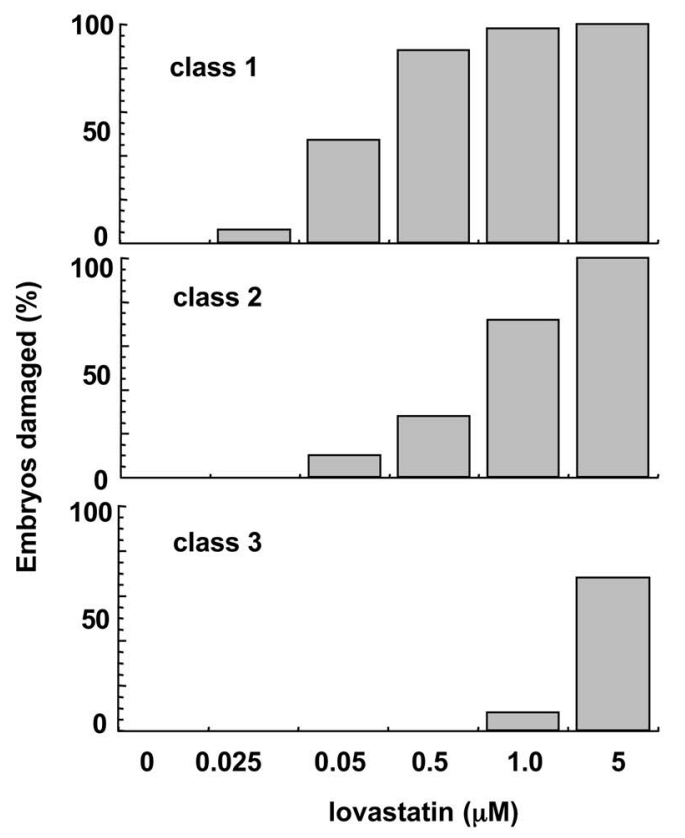

Figure 5

Lovastatin treatment disrupts myofiber structure in zebrafish embryos. (A) Zebrafish embryos (20 hpf) were treated with concentrations of lovastatin ranging from 0.025 to $5.0 \mu \mathrm{M}$ for 12 hours. Embryos were fixed and stained with antimyosin heavy chain antibody (F59) as described in Methods. Representative somite phenotypes are shown. All panels are side views, anterior, left. Original magnification, $\times 200$. (B) Quantitation of muscle damage. Morphological phenotypes shown in A were grouped into 3 classes: class 1 changes include bowing, gap formation, and blocked/disrupted fibers; class 2 changes include irregular fibers and diffuse appearance; class 3 changes are typified by irregular somite boundaries. Percentage of embryos displaying specific class defects as a function of lovastatin concentration are shown. Numbers of embryos quantitated are $151,178,163,185,189$, and 180 for the lovastatin concentrations of $0,0.025,0.05,0.5,1.0$, and $5 \mu \mathrm{M}$, respectively.

(Figure 7, D-F). Interestingly, the muscle defects caused by the z-HMG-CoA reductase knockdown were also significantly reduced in the $\mathrm{z}$-atrogin-1 knockdown (Figure 6B). These data demonstrate that the role of atrogin- 1 in mediating the effects of lovastatin is by pathways dependent on HMG-CoA reductase function.

FoxO3 activity is suppressed following lovastatin treatment. Prior studies have shown that suppression of IGF-1/PI3K/AKT signaling leading to dephosphorylation, nuclear translocation, and activation of FoxO3 are key events in atrogin-1 induction (45). We therefore examined the effects of statin administration on this pathway in muscle cell culture and in zebrafish. Treatment of C2C12 myotubes with lovastatin led to a dose-dependent reduction of phosphorylated signaling intermediates, including phospho-AKT, phospho-FoxO3, and phospho-p70S6K (Figure 8A). We then measured the effect of lovastatin on FoxO-dependent activation of the atrogin-1 promoter in zebrafish embryos. Embryos were injected with a proximal fragment of the atrogin-1 promoter linked to luciferase or the same fragment with the FoxO sites mutated (45). $0.5 \mu \mathrm{M}$ lovastatin stimulated the reporter luciferase activity more than 7-fold while stimulating the FoxO-less reporter only 3-fold (Figure 8B). Taken together, these studies suggest that, as in conditions of muscle atrophy, statin-induced atrogin-1 transcription is mediated by FoxO dephosphorylation and activation. Since lovastatin treatment still led to a small amount of luciferase activity even in the absence of FoxO-binding sites in the mutated atrogin-1 promoter reporter, additional signaling pathways may also be important in mediating the effects of statins in muscle.

$P G C-1 \alpha$ prevents muscle damage by lovastatin. Since atrogin- 1 is strongly activated following lovastatin treatment and in other models of muscle atrophy PGC- $1 \alpha$ expression both prevents atrogin- 1 induction and reduces atrophy (42), we assessed the effects of PGC-1 $\alpha$ expression on statin-induced muscle injury in zebrafish. Injection of cDNA bearing PGC- $1 \alpha$ into zebrafish embryos led to robust protein expression (Supplemental Figure 3) and dramatically prevented muscle damage by lovastatin (Figure 9, A and B). Expression of PGC-1 $\alpha$ in zebrafish embryos completely inhibited the lovastatin-induced expression of zebrafish atrogin-1 protein (Figure 9C) and protected from fiber-size reduction (Figure 9D). To monitor mitochondria during these treatments, cells from embryos exposed to lovastatin and overexpressing PGC-1 $\alpha$ were incubated with a fluorescent dye taken up by functional mitochondria (MitoTracker; ref. 49). Fluorescence intensity of zebrafish cells following treatment with lovastatin was shifted to the left, signifying less mitochondria function or content in these cells (Figure 9E and Supplemental Figure 4A). Interestingly, cells overexpressing PGC-1 $\alpha$ were significantly more fluorescent and were less affected by lovastatin treatment (Figure 9F and Supplemental Figure 4B). Expression of PGC-1 $\alpha$ in cultured muscle cells using adenoviral vectors also protected from lovastatin toxicity (Figure 10A). 


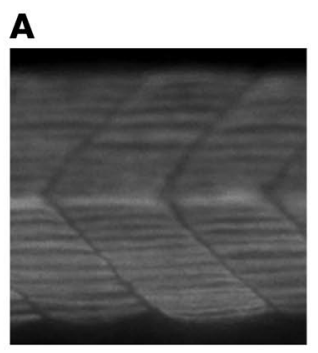

control

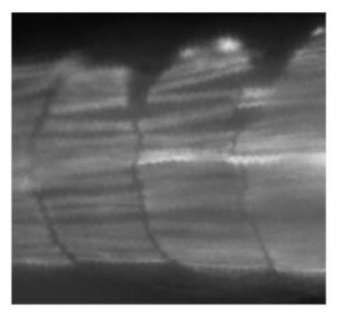

HMG CoA reductase KD

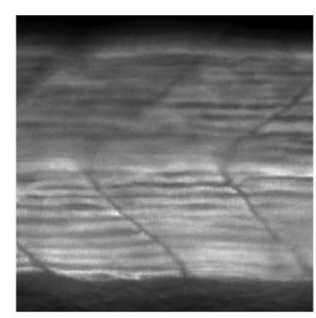

HMG CoA reductase $K D+A T-1 K D$

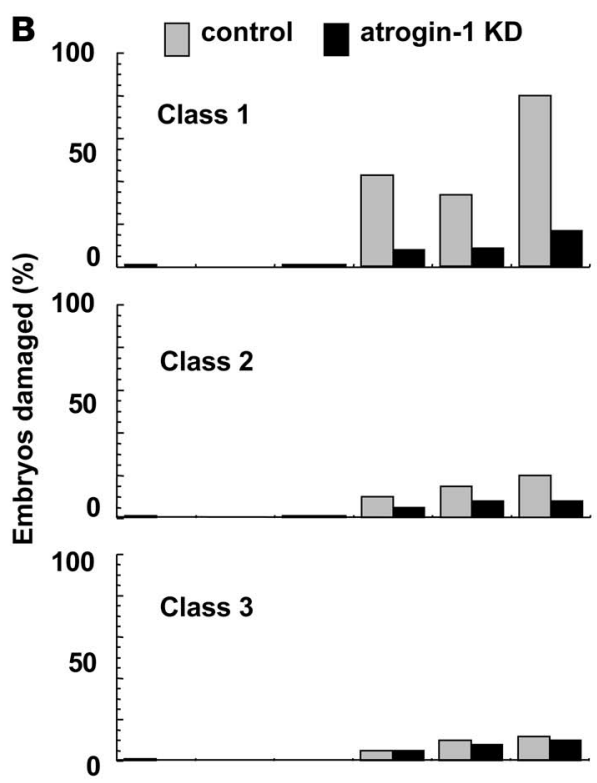

(-) MS1 MS2 MO1 MO2 Both HMG CoA reductase knockdown

\section{Figure 6}

Targeted knockdown of zebrafish HMG-CoA reductase has a muscle phenotype similar to that with lovastatin treatment and can be rescued by zebrafish atrogin-1 knockdown. (A) Myosin heavy chain staining of representative control, HMG-CoA reductase, and combined HMG-CoA reductase/atrogin-1 knockdown in zebrafish embryos. Original magnification, $\times 200$. (B) Quantitation of muscle damage. Classes of morphological phenotypes are as described in Figure 5 legend. MS1 and MS2 are missense controls for morpholinos MO1 and MO2, respectively. Note that in each case, the injection of morpholinos against atrogin-1 almost completely abolishes the damage caused by HMG-CoA reductase knockdown. Number of embryos quantitated in each bar are 231, 182, 197, and 202 for control, and 220, 204, 240, and 215 for the atrogin-1 knockdown.

In the presence of PGC- $1 \alpha, 5 \mu \mathrm{M}$ lovastatin caused almost no change in myotube integrity or size. Likewise, PGC- $1 \alpha$ completely suppressed atrogin- 1 induction in these cultures while inducing expression of mitochondrial oxidative phosphorylation genes (Figure 10B). Taken together, these experiments show that as in models of atrophy, PGC-1 $\alpha$ expression protects against muscle damage, presumably by suppressing FoxO function (42) and augmenting mitochondrial number and/or function.

\section{Discussion}

Through a combination of complementary in vitro, animal, and human experiments, this report provides the first evidence that statins induce muscle damage by induction of the muscle-specific E3 ubiquitin ligase atrogin-1. Since little was known about the mechanisms by which statins induce muscle damage, we hypothesized that statin toxicity might share features of an atrophic response seen in other muscle-wasting disease states. In these conditions, a common series of transcriptional changes is found in muscle (20). Coordinated regulation of atrophy-related genes (atrogenes), including atrogin-1, promotes increased muscle protein degradation by the ubiquitin proteasome pathway. This study on the induction and functional role of atrogin- 1 following statin administration suggests that important features of the atrophic response are also present in this form of muscle injury. Not only does statin treatment lead to increased atrogin-1 expression, but rates of protein breakdown are also increased in lovastatin-treated muscle cells. Furthermore, the experiments shown here and performed by others (50) demonstrate suppression of IGF-1 signaling after statin treatment. This suggests a common mechanism for the induction of atrogin-1 following statin treatment and in muscle atrophy, where suppression of IGF-1 signaling leads to FoxO dephosphorylation, nuclear localization, and transcription of the atrogin-1 gene (45). Interestingly, atrogin-1 is not simply a marker of muscle atrophy and damage, as its expression has not been shown to be affected in various stages of Duchenne muscular dystrophy (51). Though the fact that lovastatin-induced muscle damage is markedly reduced in atrogin-1 knockout cells suggests a direct mechanistic role for atrogin- 1 in statin myopathy, identification of atrogin- 1 targets and function will ultimately be required to fully understand the process. We are now exploring whether the expression of other atrogenes, for example MuRF-1, another muscle-specific ubiquitin ligase, is also induced during statin muscle toxicity.

Statin toxicity in skeletal muscle could also, in principle, be the result of other processes including necrosis or autophagy. Light microscopic findings in patients with statin myopathy are variable and not pathognomonic (52), but muscle from statin-treated rats often demonstrates mitochondrial damage and multilamellar structures suggestive of autophagic vacuole formation (53, 54). Indeed, recent data suggest that atrophying muscle generally activates autophagic proteolytic pathways (J. Zhao, A. Goldberg, and M. Sandri, unpublished observations).

The role of atrogin-1 in mediating statin muscle toxicity is not yet clear. As a ubiquitin protein ligase, it likely targets for degradation 1 or a set of key muscle proteins important for maintaining myofiber integrity. Tintgnac and colleagues have reported MyoD, the transcriptional activator critical for muscle development, as an atrogin-1 target (55); however, atrogin-1 knockout animals develop adult muscle normally, and MyoD levels actually rise upon 
A

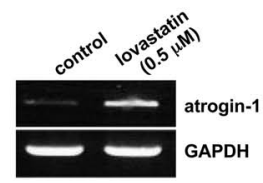

B

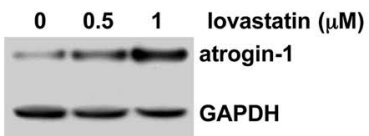

D

$$
0 \mu \mathrm{M}
$$
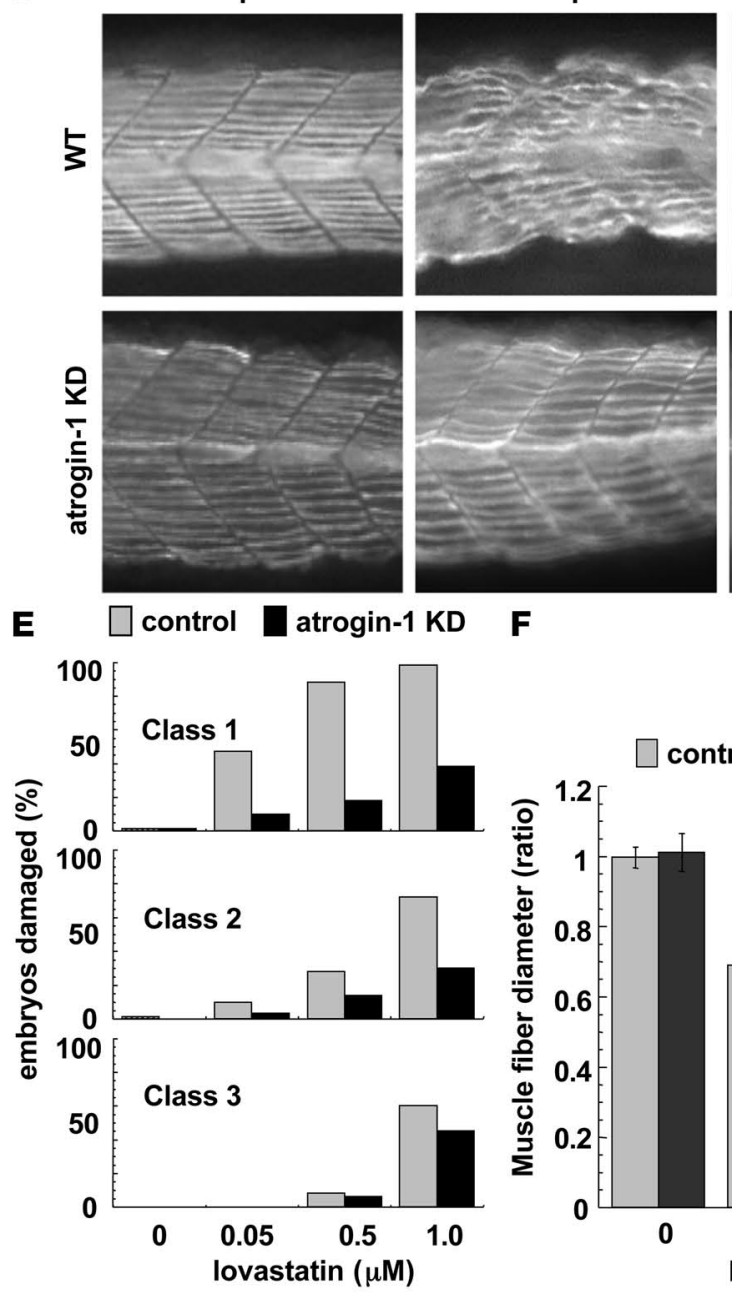

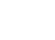

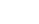

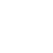

C

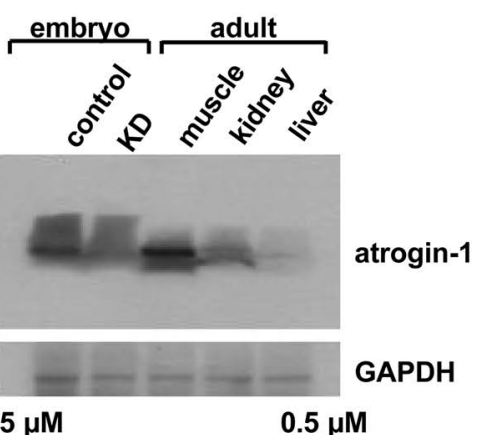

$\mathbf{F}$
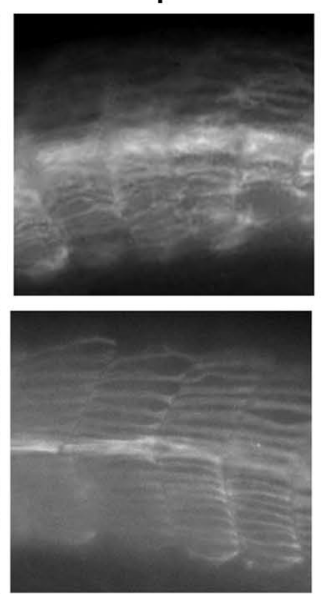

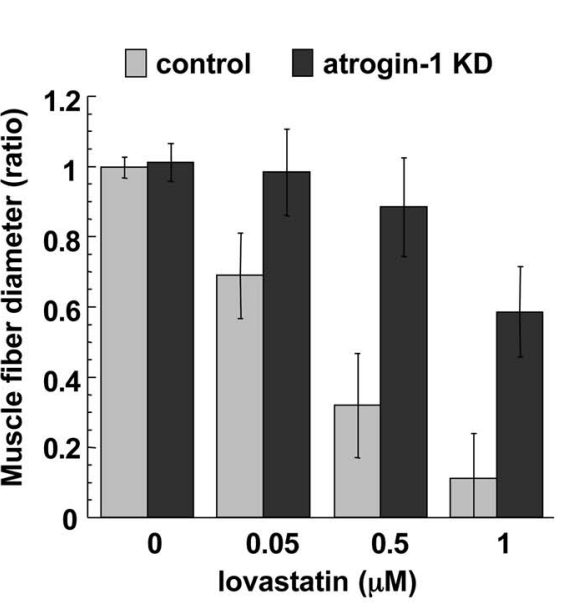

\section{Figure 7}

Atrogin-1 knockdown reduces lovastatin-induced muscle damage in zebrafish embryos. Zebrafish embryos (20 hpf) were treated with lovastatin $(0.5 \mu \mathrm{M})$ for 12 hours. Atrogin-1 mRNA was measured by PCR $(\mathbf{A})$ and zebrafish atrogin-1 protein by Western blotting (B). Five embryos were used for each analysis. (C) Western blot probed for atrogin-1 using protein lysates $(20 \mu \mathrm{g} / \mathrm{lane})$ derived from control zebrafish embryos, zebrafish embryos injected with morpholino against atrogin-1, adult zebrafish muscle, adult zebrafish kidney, and adult zebrafish liver. Five embryos were used for each analysis. (D) Myosin heavy-chain staining of representative control and atrogin-1 knockdown embryos. Note that suppression of atrogin-1 protects from statininduced damage. Original magnification, $\times 200$. (E) Quantitation of muscle damage. Classes of morphological phenotypes are as described in Figure 5 legend. Note that at each lovastatin concentration, the injection of morpholinos against atrogin-1 almost completely abolishes the damage caused by lovastatin. Numbers of embryos quantitated are 235, 182, 197, and 202 for the controls and 220, 204, 240 , and 215 for the atrogin-1 knockdowns at lovastatin concentrations of $0,0.05,0.5$, and $1.0 \mu \mathrm{M}$, respectively. (F) Muscle fiber diameter was measured following myosin heavy-chain staining as described in Methods. At least 500 fibers were measured at each lovastatin concentration. Results were graphed as the ratio of mean experimental fiber size $\pm \mathrm{SEM} /$ mean control fiber size \pm SEM. Control fiber size: $7.60 \pm 0.19 \mu \mathrm{M}$. denervation instead of fall. For these reasons, MyoD is unlikely to be a key physiological substrate that can explain the development of muscle atrophy or statin toxicity. Likewise, Li and coworkers recently found calcineurin in cardiac cells to be degraded in an atrogin-1-dependent manner (25); however, atrogin-1 knockout animals show no cardiac abnormalities or tendency toward cardiac hypertrophy, and in skeletal muscle, calcineurin signaling seems to affect fiber type more than fiber size (56).

It has been unclear whether the effects of statin administration are a consequence of HMG-CoA reductase inhibition or another nonspecific effect of the agents. The fact that structurally diverse statins can produce myopathy in patients suggests that toxicity is the result of inhibition of this enzyme. Our studies in fish, in which knockdown of the HMG-CoA reductase gene leads to a muscle phenotype closely resembling that seen following statin treatment, strongly argues that statin-induced muscle toxicity is indeed the result of HMG-CoA reductase inhibition. Nevertheless, it is clear that the pharmacodynamics of these agents, for example, their muscle penetration and volume of distribution, can result in varying muscle toxicity profiles in any given patient. Some statins, such as cerivastatin, have especially marked toxicity in skeletal muscle and have been removed from clinical use $(1,4)$. It has been suggested that more hydrophilic inhibitors, e.g., pravastatin, may be less toxic $(14,57)$; however, other studies have not found a clear connection between lipophilicity and toxicity $(58,59)$. Several nitrogencontaining bisphosphonates, such as alendronate and risedronate, 
A

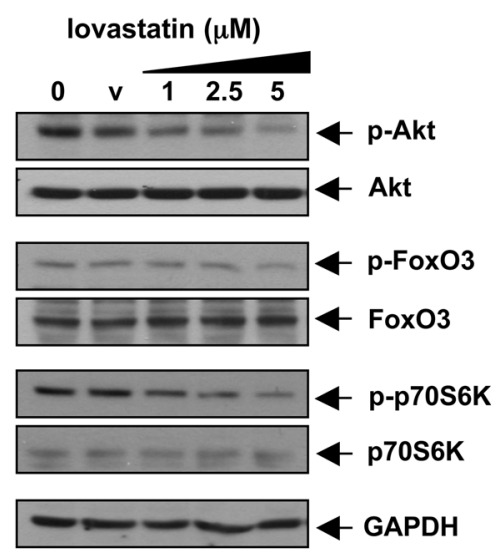

B

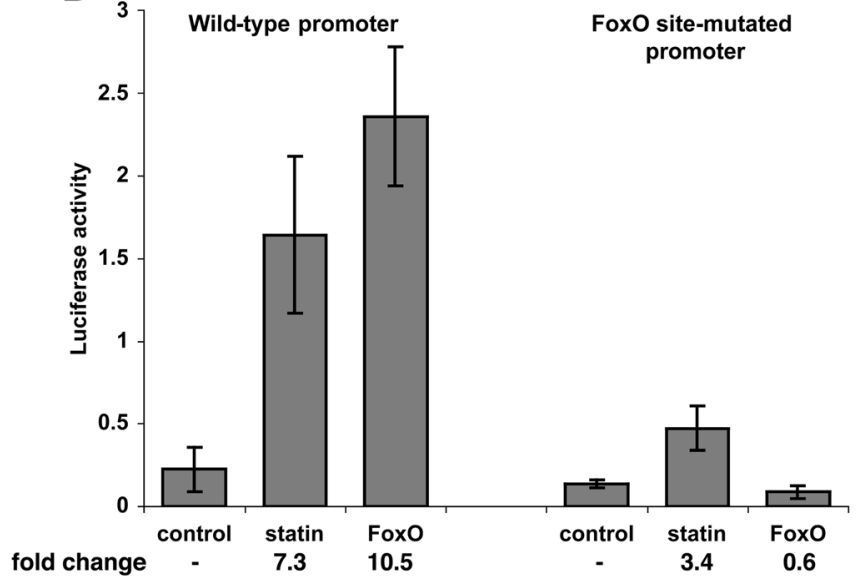

Figure 8

Lovastatin suppresses IGF-1 signaling. (A) Lovastatin suppresses PI3K/AKT/FoxO signaling in C2C12 myotubes. C2C12 myotubes were treated for 24 hours with vehicle $(\mathrm{v})$ or lovastatin at the indicated concentrations. Protein lysates were prepared and subjected to immunoblot analysis with various antibodies. (B) Lovastatin induces the atrogin-1 promoter in a FoxO-dependent manner in zebrafish embryos. Embryos at the 1-cell stage were injected with the $0.4-\mathrm{kb}$ atrogin-1 promoter reporter with the FoxO sites present or mutated (45). Embryos were grown in the presence of $0.5 \mu \mathrm{M}$ lovastatin and luciferase activity measured in embryo lysates 48 hours later. Constitutively active FoxO3a was coinjected as a positive control.

are also known to inhibit enzymes of the mevalonate pathway (60). These drugs might also prevent biosynthesis of isoprenylated compounds essential for posttranslational modification of key muscle proteins. It has been noted that a certain number of patients treated with these compounds exhibit severe muscle pain (61). These data also support the notion that drugs that affect the mevalonate pathway, such as HMG-CoA reductase inhibitors and this class of bisphosphonates, can induce muscle damage.

The beneficial effects of PGC- $1 \alpha$ expression in reducing statininduced skeletal muscle injury, both in cell culture and in zebrafish, suggest that either mitochondrial number or improved mitochondrial function may be central to maintaining muscle integrity. Indeed, compromised mitochondrial function has been linked to muscle atrophy associated with many catabolic states. Insulin resistance in skeletal muscle correlates with decreased mitochondrial oxidative capacity and ATP synthesis and decreased expression of genes that control mitochondrial activity $(62,63)$. As in models of muscle atrophy, PGC- $1 \alpha$ expression also prevents atrogin- 1 induction following statin exposure by suppressing expression or function of forkhead transcription factors that are critical to the induction of atrogin- $1(42,45)$. Our results suggest that other agents that increase PGC- $1 \alpha$ action or activity, for example, metformin, might be useful in preventing or countering the detrimental effects of statins in muscle. The observation that statin myotoxicity seems to affect type II, glycolytic myofibers more than type I, oxidative fibers $(54,64,65)$ could be explained by the protective effect of PGC-1 $\alpha$ expression and mitochondrial density, which is greater in oxidative fibers. On the other hand, endurance exercise, which also increases the expression of PGC- $1 \alpha$, seems to increase the likelihood of toxic reactions to statin therapy in clinical practice (66-68).

About a third of patients with statin-induced myopathy appear to respond to high-dose $\mathrm{CoQ}_{10}$ administration (P.S. Phillips, unpublished observations). $\mathrm{CoQ}_{10}$ is a critical component of the oxidative phosphorylation machinery that resides in the mitochondrial inner membrane. HMG-CoA reductase activity is required for endogenous $\mathrm{CoQ}_{10}$ synthesis since the molecule is prenylated.
Others have reported that, in vitro, statins inhibit geranylgeranylation (69), and so it is tempting to speculate that this arm of the HMG-CoA reductase biosynthetic pathway is required to maintain muscle function. PGC-1 $\alpha$ expression leading to increased mitochondrial mass might then reduce the effect of statins by increasing the pool of mitochondrial $\mathrm{CoQ}_{10}$ and placing less of a requirement on new (statin-inhibitable) $\mathrm{CoQ}_{10}$ production.

Patients with statin-induced muscle injury represent a heterogeneous group, ranging from those with overt muscle necrosis (rhabdomyolysis) to those with muscle pain and no serum creatine kinase elevation. They also present variably clinically, and the resolution of their symptoms after cessation of statin therapy can be variable in time course as well. It is conceivable that polymorphisms in the atrogin-1 gene or promoter might explain differences in susceptibility, clinical presentation, or course in patients with statin myopathy. To explore this further, it will be important to better define the transcription factors and cofactors such as PGC- $1 \alpha, \mathrm{NF}-\mathrm{\kappa B}$, and FoxO that regulate atrogin-1 expression as well as the upstream signaling pathways that impinge on them and identify how statins affect these events. Such studies should shed additional light on the molecular mechanisms of statin-induced myopathy and enable the stratification of patients who might be susceptible to such injury.

\section{Methods}

Plasmids, viral constructs, antibodies, and signaling inbibitors. Polyclonal antiatrogin-1 antibody was used as described (26). Antibodies directed against FoxO3 and phosphorylated FoxO3 were from Upstate Biotechnology. Rabbit polyclonal anti-phospho-AKT (Ser473) and anti-phospho-p70S6K (Thr389), anti-AKT, and anti-p70S6K were purchased from Cell Signaling. Anti-GAPDH antibodies were from Santa Cruz Biotechnology Inc.

Muscle biopsies. Muscle was obtained from 3 groups of patients whose characteristics are detailed in Supplemental Table 1. The statin-treated group included 4 subjects with statin-induced myopathy and 4 with statin-induced rhabdomyolysis as commonly defined (6). The nonstatin myopathy group included 6 statin-naive subjects with undefined myopathy. Both of these groups underwent percutaneous muscle biopsies of the vastus lateralis muscle 
A

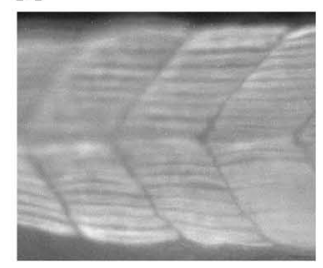

control

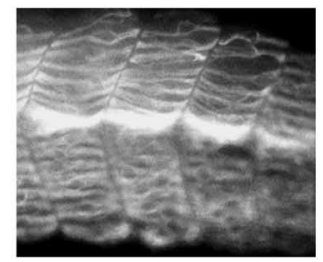

lovastatin

C

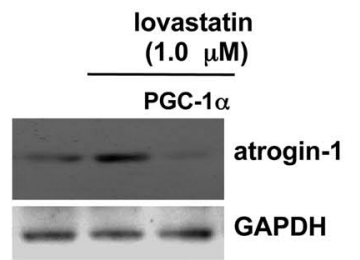

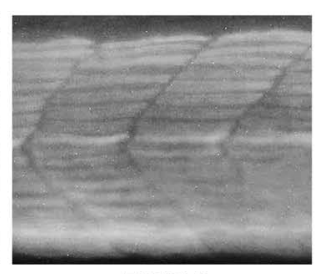

PGC-1 $\alpha$

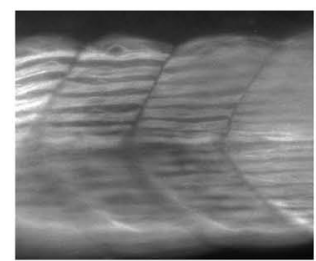

lovastatin + PGC-1 $\alpha$

D

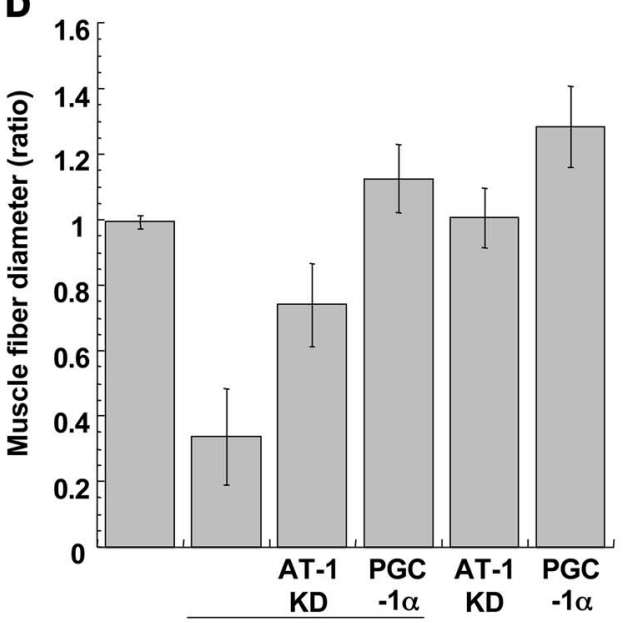

lovastatin $(0.5 \mu \mathrm{M})$

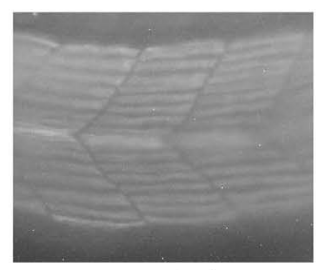

control

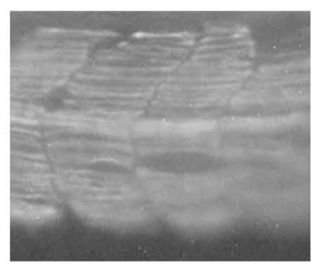

PGC-1 $\alpha$ KD
B

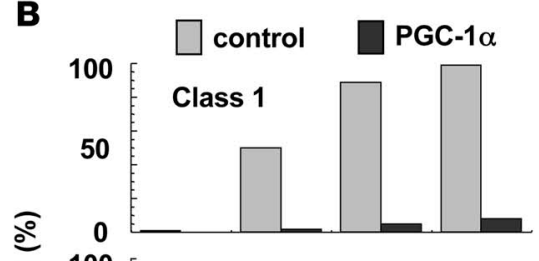

Class 2

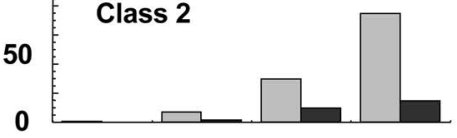

100

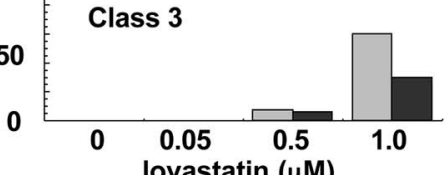

E
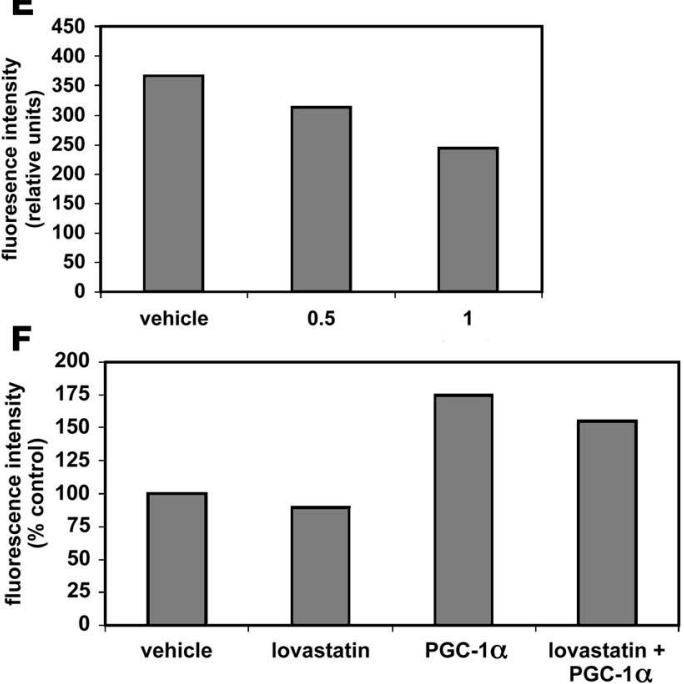

Figure 9

PGC-1 $\alpha$ expression reduces lovastatin-induced atrogin-1 expression and muscle damage in zebrafish embryos. (A) Myosin heavy chain staining of representative zebrafish embryo somites following injection of $100 \mathrm{pg}$ PGC- $1 \alpha$ cDNA or vehicle in the presence or absence of $0.5 \mu \mathrm{M}$ lovastatin for 12 hours (left box) or morpholino oligonucleotides against z-PGC-1 $\alpha$ (right box). Note that suppression of atrogin-1 protects from statininduced damage and that suppression of PGC- $1 \alpha$ has a similar muscle phenotype as lovastatin treatment. Original magnification, $\times 200$. (B) Quantitation of muscle damage. Classes of morphological phenotypes are as described in Figure 5. Note that at each lovastatin concentration, the injection of PGC-1 $\alpha$ cDNA almost completely abolishes the damage caused by lovastatin. Number of embryos quantitated are $137,112,139$, and 122 for the controls and 120,103,108, and 107 for the PGC- $1 \alpha$-injected embryos at the lovastatin concentrations of $0,0.05,0.5$, and $1.0 \mu \mathrm{M}$, respectively. (C) Western blot of atrogin-1 following $0.5 \mu \mathrm{M}, 12$ hours lovastatin treatment in zebrafish embryos injected or not with 100 pg PGC-1 $\alpha$ cDNA. (D) Muscle fiber diameter was measured following myosin heavy chain staining as described in Methods in embryos injected with atrogin-1 morpholinos or PGC-1 $\alpha$ cDNA. At least 500 fibers were measured at each lovastatin concentration. Results were graphed as the ratio of mean experimental fiber size $\pm \mathrm{SEM} /$ mean control fiber size $\pm \mathrm{SEM}$. Control fiber size: $7.58 \pm 0.10 \mu \mathrm{M}$. (E) Mitochondrial function is diminished by lovastatin treatment of zebrafish embryos. Cells from zebrafish embryos treated with varying concentrations of lovastatin $(0-1 \mu \mathrm{M})$ were stained with MitoTracker, and fluorescence intensity, reflecting mitochondrial function, was detected by fluorescence-activated cell sorting. Representative data of mean fluorescence intensity from 3 independent experiments are shown. (F) PGC-1 $\alpha$ augments mitochondrial staining and protects against lovastatin's effects in zebrafish embryos. Embryos were injected with PGC-1 $\alpha$ cDNA as described in Methods, then treated with lovastatin $(0.5 \mu \mathrm{M})$ for 24 hours. As in E, dispersed embryonic cells were stained with MitoTracker and detected by FACS. Data are presented as percentage of mean fluorescence intensity in vehicle-treated embryos.

using a Bergstrom needle. The control group included 5 statin-naive subjects who volunteered muscle at the time of knee arthroplasty. Muscle from subjects in all 3 groups was snap-frozen in liquid nitrogen for subsequent analysis. All subjects signed a consent form approved by the institutional review board of Scripps Mercy Hospital.
Cell culture. Mouse myoblast cell line $\mathrm{C} 2 \mathrm{C} 12$ was purchased from ATCC and maintained in DMEM containing 10\% fetal bovine serum (HyClone) and penicillin $(100 \mathrm{U})$ and streptomycin $(50 \mu \mathrm{g} / \mathrm{ml})$ (Invitrogen). When C2C12 cells reached $90 \%$ confluence, medium was replaced with differentiation medium of DMEM supplemented with $2 \%$ horse serum (ATCC) to induce 
A

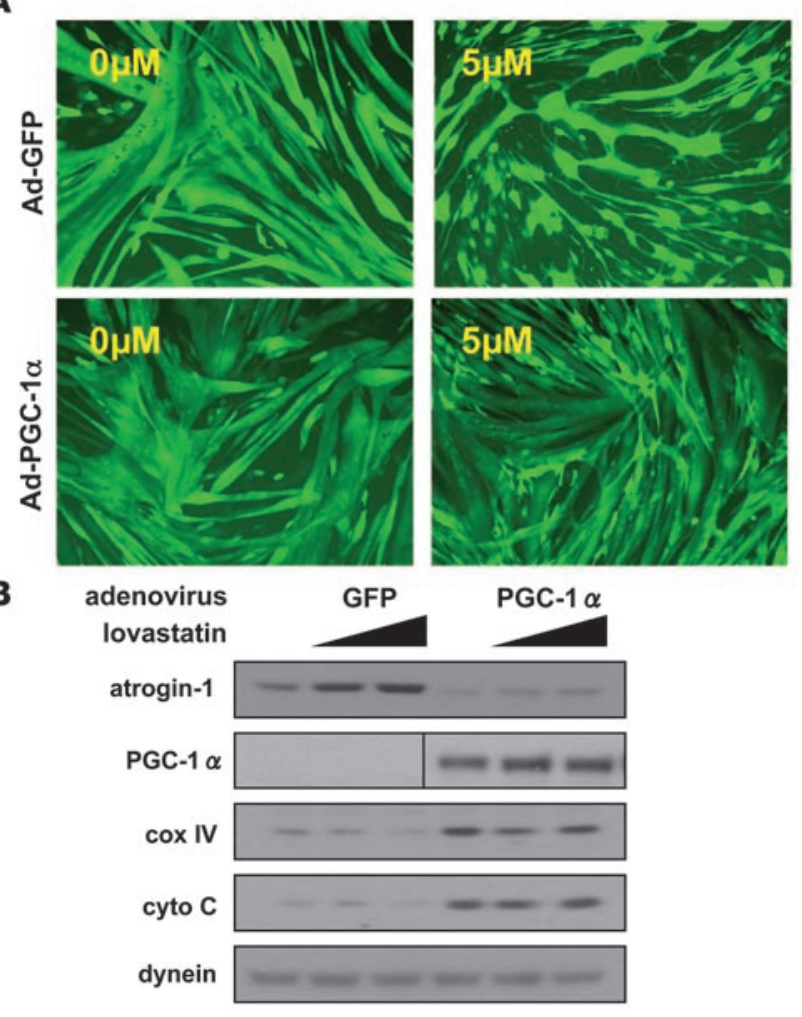

myotube formation. Cells were used for experiments in 4-5 days after differentiation. Lovastatin (>98\% purity) (mevinolin, Sigma-Aldrich) was prepared as a $50-\mathrm{mM}$ stock solution in DMSO as reagent vehicle, further diluted in DMSO, and added into the medium. The final volume DMSO in medium was not more than $0.125 \%$, and no obvious cytotoxicity was observed. Equal volume of reagent vehicle was used for all experiments, and reagent vehicles only served as controls. Each experiment was performed at least 3 times. Primary mouse myoblasts from atrogin-1 null mice (Regeneron) were isolated as follows: muscle was removed from the hind limbs of 2-week-old mice. After treatment with $0.1 \%$ collagenase D and Dispase II (Roche), the isolated cells were plated on collagen-coated (type I, Roche) dishes. Myoblasts were subsequently enriched and cultured in F-10 nutrient medium with $20 \%$ fetal calf serum and $2.5 \mathrm{ng} / \mathrm{ml} \mathrm{bFGF} \mathrm{(Invitrogen).} \mathrm{Myotubes} \mathrm{were} \mathrm{induced} \mathrm{in} \mathrm{dif-}$ ferentiation medium. All media contained $1 \times$ Primocin (InvivoGen). The cultures were maintained at $37^{\circ} \mathrm{C}$, under $5 \%$ and $8 \% \mathrm{CO}_{2}$ air humidified atmosphere for myoblasts and myotubes, respectively. Cultures were ready to use in assays on day 2 in differentiation medium when the myotubes had formed and were contracting. The present experiments using mice were reviewed and approved by the Institutional Animal Care and Use Committee of Beth Israel Deaconess Medical Center and Harvard Medical School.

Myotube fiber size. Size was quantified by measuring a total of 200 tube diameters as described in ref. 45. In brief, muscle fiber size from 4 random fields at $\times 100$ magnification was measured using IMAGE software (Scion). All data were expressed as mean \pm SEM. Comparisons were made by using Student's $t$ test, with $P<0.05$ considered statistically significant.

Quantitative PCR. Atrogin-1 mRNA levels were determined by real-time PCR using the Applied Biosystems 7500 real-time PCR analyzer according to the method recently described by others $(70,71)$. Multiplexed amplification reactions were performed using $18 \mathrm{~S}$ rRNA as an endogenous control (18S rRNA primers/VIC-labeled probe; Applied Biosystems, 4310893E) using the TaqMan One Step PCR Master Mix reagents Kit (4309169,

\section{Figure 10}

PGC-1 $\alpha$ reduces lovastatin-induced atrogin-1 expression and muscle damage in $\mathrm{C} 2 \mathrm{C} 12$ myotubes. (A) $\mathrm{C} 2 \mathrm{C} 12$ myotubes infected for 68 hours with control adenovirus or adenovirus bearing PGC- $1 \alpha$ were visualized by GFP. $5 \mu \mathrm{M}$ lovastatin or vehicle was present for the final 48 hours. Original magnification, $\times 100$. (B) Western blot for atrogin-1 in controlinfected or PGC-1 $\alpha$-infected $\mathrm{C} 2 \mathrm{C} 12$ myotube cultures in the presence of increasing concentrations of lovastatin $(0-5 \mu \mathrm{M})$ for 48 hours. Expression of mitochondrial electron transport proteins cytochrome oxidase IV and cytochrome $c$ were monitored by immunoblot. Note that lovastatininduced atrogin-1 expression in the presence of PGC-1 $\alpha$ is suppressed.

Applied Biosystems). The following settings were used: stage 1 (reverse transcription), $48^{\circ} \mathrm{C}$ for 30 minutes; stage 2 (denaturation), $95^{\circ} \mathrm{C}$ for 10 minutes; and stage 3 (PCR), $95^{\circ} \mathrm{C}$ for 15 seconds and $60^{\circ} \mathrm{C}$ for 60 seconds for 40 cycles. The sequences of the forward, reverse, and double-labeled oligonucleotides for atrogin-1 were as follows: forward, 5'-CTTTCAACAGACTGGACTTCTCGA-3'; reverse, 5'-CAGCTCCAACAGCCTTACTACGT-3'; TaqMan probe, sequence, 5'- FAM-TGCCATCCTGGATTCCAGAAGATTCAAC-TAMRA-3'. Fluorescence data were analyzed by SDS1.7 software (Applied Biosystems). The $\mathrm{C}_{\mathrm{t}}$ (threshold cycle) values for each reaction were transferred to a Microsoft Excel spreadsheet, and calculation of relative gene expression was performed from these data according to published algorithms (TaqMan Cytokine Gene Expression Plate 1 protocol, Applied Biosystems). All RNA samples were analyzed in triplicate, with the mean value used in subsequent analyses.

Western blotting. Cultured cells after treatment were collected at specific times and solubilized in RIPA lysis buffer: Tris- $\mathrm{HCl}(50 \mathrm{mM}), \mathrm{pH} 7.4, \mathrm{NaCl}$ $(150 \mathrm{mM}), \mathrm{NP}-40$ (1\%), sodium deoxycholate $(0.5 \%)$, SDS $(0.1 \%)$ (Boston Bioproducts), protease (Roche), and phosphatase (Sigma-Aldrich) inhibitor cocktail. Proteins were separated by SDS-PAGE, transferred to PVDF membranes, and visualized by Western blotting using alkaline phosphatase-based CDP-star chemiluminescent detection according to the manufacturer's protocol (Applied Biosystems). Zebrafish embryos were homogenized in SDS sample buffer (30 embryos/30 $\mu$ l of sample buffer) with microfuge pestle until the lysate became uniform in consistency and a stringy mass was no longer observed. The lysate was boiled for 5 minutes, and centrifuged supernatant was processed for Western blotting (72).

Measurement of proteolytic rate. Differentiated C2C12 myotubes were incubated with ${ }^{3} \mathrm{H}$-tyrosine $(5 \mu \mathrm{Ci} / \mathrm{ml}$ media) for 20 hours to label cell proteins and switched to medium containing $2 \mathrm{mM}$ unlabeled tyrosine, vehicle, lovastatin, or dexamethasone for another 20 hours. After replacement, an aliquot of medium was collected once per hour for 4 hours. The collected media was treated with TCA $(10 \% \mathrm{w} / \mathrm{v}$ final concentration) to precipitate protein. Since the high concentration of unlabeled tyrosine in the media prevents reincorporation of ${ }^{3} \mathrm{H}$-tyrosine into new protein, the radioactivity in the supernatant represents degraded protein from the pool of prelabeled, intracellular, long-lived proteins. Proteolytic rate was defined as the percentage of released radioactivity per hour calculated from the period of 20 hours to 24 hours of lovastatin or dexamethasone treatment, when ${ }^{3} \mathrm{H}$-tyrosine release is linear over time. These rates were compared with proteolytic rates in parallel cultures treated with vehicle alone. All measurements were done in triplicate and then independently repeated at least twice.

Zebrafish lines and maintenance. Adult zebrafish (Danio rerio) were maintained as described under standard laboratory conditions at $28.5^{\circ} \mathrm{C}$ in a 14-hour light/10-hour dark cycle (73) Developmental stages were determined by embryo morphology and $h p f(74)$. To examine the effects of statin, the embryos at 20-24 hpf were immersed in the embryonic water $(500 \mu \mathrm{M}$ $\left.\mathrm{NaCl}, 170 \mu \mathrm{M} \mathrm{KCl}, 330 \mu \mathrm{M} \mathrm{CaCl}_{2}, 330 \mu \mathrm{M} \mathrm{MgSO}_{4}\right)$ at a concentration of $0.005-10 \mu \mathrm{M}$ of lovastatin (mevinolin; Sigma-Aldrich) including $0.003 \%$ 
1-phenyl-2-thiourea (Sigma-Aldrich) to inhibit pigmentation in 24-well plate. After $32 \mathrm{hpf}$, the embryos were fixed by $4 \%$ paraformaldehyde in PBS.

Antibody staining. Whole zebrafish staining: zebrafish embryos were fixed by $4 \%$ paraformaldehyde in PBS overnight. After fixation, the embryos were washed by $\mathrm{PBS}$ and stored for at least 1 hour at $-20^{\circ} \mathrm{C}$ in methanol and permeabilized for 30 minutes at $-20^{\circ} \mathrm{C}$ in acetone. Embryos were incubated with blocking buffer (1\% BSA, 0.1\% Tween-20 in PBS), and incubated with diluted primary antibody, anti-slow twitch myosin F59 (1: 200; Developmental Studies Hybridoma Bank [DSHB], Department of Biological Sciences, University of Iowa) $(75,76)$ in blocking solution overnight at $4^{\circ} \mathrm{C}$. Staining was detected by using goat anti-mouse TRITC secondary antibody (1:200; Southern Biotechnology Associates) in blocking solution for 4 hours at room temperature (48). For cross-sectional staining, embryos were fixed overnight in $4 \%$ paraformaldehyde (PFA) and cryoprotected by overnight incubation with increasing concentrations of sucrose (up to $30 \%$ ). Samples were embedded in OCT compound and then equilibrated to $-80^{\circ} \mathrm{C}$. Sections $(10-\mu \mathrm{m}$ thick) were collected on SuperFrost/Plus slides and dried. Sections were rehydrated in PBS and blocked for 1 hour in blocking buffer (1\% BSA, 0.1\% Tween in PBS). Sections were incubated overnight at $4^{\circ} \mathrm{C}$ with primary antibody diluted in blocking buffer. Sections were stained with antibody as above.

Antisense morpholino oligonucleotides sequence and injection. Morpholino antisense oligonucleotides (MOs) were designed and synthesized by Gene Tools LLC. MOs were used for knockdown of the z-atrogin-1, z-HMGCoA reductase. The sequences are 5'-TTGTCCAAGAAACGGCATTGCAAG-3' for targeting ATG sequence of $z$-atrogin-1 and $5^{\prime}$-AAAGCCACCATCATGTACCTGTCTG-3' for targeting splicing sequence of $z$-atrogin-1; 5'-ATTCGGAAAAGTCTCGTCAGCATGG-3' and 5'-GTGATGACCTGCTCAATGTCCATGC-3' for targeting ATG sequences of $\mathrm{z}$-HMGCoA reductases; 5'-ATTCCGAAAACTCTCCTCACGATGG- $3^{\prime}$ and $5^{\prime}$ - GTCATCACCTCCTCAATCTCCATCC-3' for control 5-mispair MOs; and 5'-CCTGATTACACCTGTCCCACGCCAT-3' and 5'-CCAGCCCCTGAATGACGGCACGGAA- $3^{\prime}$ for targeting ATG sequences of z-PGC-1 $\alpha$. For injections, MOs were diluted to $250 \mu \mathrm{M}$. MOs were injected at $4 \mathrm{ng}$ per embryo into 1 cellstage embryos at the yolk and cytoplasm interface.

In vivo atrogin-1 promoter reporter assay. Zebrafish embryos (20 per condition) were injected with a mixture of DNA including the atrogin-1 promoter luciferase construct (45) and a control thymidine kinase promoter sea pansy luciferase construct (50 pg total DNA/embryo, 25:1 ratio) with or without 50 pg constitutively active FoxO3a as a positive control (45). Total injected cDNA was adjusted to 100 pg per embryo with empty vector cDNA. Half the embryos not coinjected with caFoxO3a were incubated in water containing lovastatin $(0.5 \mu \mathrm{M})$. Injections were performed at the 1 cell-stage at the yolk-cytoplasm interface. Embryos were then homogenized in Passive Lysis Buffer (Promega) at 48 hpf. The luciferase activity was determined using a luminometer normalized using sea pansy luciferase activity under the control of the thymidine kinase promoter.

Mitochondrial staining and FACS analysis. Embryos were treated with lovastatin $(0.0 .5,1.0 \mu \mathrm{M}$, at $20-32 \mathrm{hpf})$ or treated with the combination of lovastatin $(0.5 \mu \mathrm{M})$ following PGC-1 $\alpha$ (or vehicle) cDNA injection (100 pg/ embryo, at 1-cell stage). $0.003 \%$ 1-phenyl-2-thiourea (Sigma-Aldrich) was added at $20 \mathrm{hpf}$. After phenotypes were observed, 100 embryos from each condition were dechorionated by protease and homogenized for 3-5 minutes in $0.9 \times \mathrm{PBS} / 10 \% \mathrm{FBS}$, then centrifuged at $1,000 \mathrm{~g}$ for 5 minutes, digested by Trypsin/EDTA, and dispersed at room temperature. After adding $1 \mathrm{ml}$ of $0.9 \times \mathrm{PBS} / 10 \% \mathrm{FBS}$, dispersed cells were filtered $(100-\mu \mathrm{m}$ pore size) and washed twice with $0.9 \times \mathrm{PBS} / 10 \% \mathrm{FBS}$. Cells were incubated in $100 \mathrm{nM}$ MitoTracker Red CMXRos (Invitrogen) in $0.9 \times \mathrm{PBS} / 10 \%$ FBS for 15 minutes in the dark. The cells were washed twice with $0.9 \times \mathrm{PBS} / 1 \% \mathrm{FBS}$ and subjected to FACS analysis. EPICS XL (Beckman Coulter) was used for fluorescence detection (absorption $578 \mathrm{~nm}$, emission $599 \mathrm{~nm}$ ), and data were analyzed with Expo32ADC software. We counted 10,000 cells for each condition.

Statistics. All values are expressed as mean \pm SEM. One-way ANOVA was used to identify significant differences in multiple comparisons. A level of $P<0.05$ was considered statistically significant.

\section{Acknowledgments}

We thank members of the Sukhatme, Karumanchi, and Goldberg laboratories for their useful discussions. We also thank Sonia Sinha and Tanner Boes for technical support. The primary myocytes lacking atrogin-1 were derived from MAFbx knockout mice kindly provided to us from Regeneron Pharmaceuticals. This work was supported by grants from the NIH (DK062307 to S.H. Lecker) and seed funds from Beth Israel Deaconess Medical Center (to V.P. Sukhatme).

Received for publication May 18, 2007, and accepted in revised form September 5, 2007.

Address correspondence to: Vikas P. Sukhatme or Stewart H. Lecker, Beth Israel Deaconess Medical Center, 330 Brookline Avenue, Boston, Massachusetts 02215, USA. Phone: (617) 667-2147; Fax: (617) 667-5276; E-mail: vsukhatm@bidmc.harvard.edu (V.P. Sukhatme); slecker@bidmc.harvard.edu (S.H. Lecker).

Jun-ichi Hanai and Peirang Cao contributed equally to this work.

Vikas P. Sukhatme and Stewart H. Lecker are co-senior authors.
1. Staffa, J.A., Chang, J., and Green, L. 2002. Cerivastatin and reports of fatal rhabdomyolysis. N. Engl. J. Med. 346:539-540.

2. Thompson, P.D., Clarkson, P., and Karas, R.H. 2003. Statin-associated myopathy. JAMA. 289:1681-1690.

3. Ballantyne, C.M., et al. 2003. Risk for myopathy with statin therapy in high-risk patients. Arch. Intern. Med. 163:553-564.

4. Graham, D.J., et al. 2004. Incidence of hospitalized rhabdomyolysis in patients treated with lipid-lowering drugs. JAMA. 292:2585-2590.

5. Phillips, P.S., et al. 2002. Statin-associated myopathy with normal creatine kinase levels. Ann. Intern. Med. 137:581-585.

6. Antons, K.A., Williams, C.D., Baker, S.K., and Phillips, P.S. 2006. Clinical perspectives of statininduced rhabdomyolysis. Am. J. Med. 119:400-409.

7. Foley, K.A., Massing, M.W., Simpson, R.J., Jr., Alexander, C.M., and Markson, L.E. 2004. Population implications of changes in lipid management in patients with coronary heart disease. Am. J. Cardiol. 93:193-195.

8. O'Meara, J.G., et al. 2004. Ethnic and sex differences in the prevalence, treatment, and control of dyslipidemia among hypertensive adults in the GENOA study. Arch. Intern. Med. 164:1313-1318.

9. Baker, S.K., and Tarnopolsky,M.A. 2001. Statin myopathies: pathophysiologic and clinical perspectives. Clin. Invest. Med. 24:258-272.

10. Baker, S.K. 2005. Molecular clues into the pathogenesis of statin-mediated muscle toxicity. Muscle Nerve. 31:572-580.

11. Liao, J.K. 2002. Isoprenoids as mediators of the biological effects of statins. J. Clin. Invest. 110:285-288. doi:10.1172/JCI200216421.

12. Flint, O.P., Masters, B.A., Gregg, R.E., and Durham, S.K. 1997. Inhibition of cholesterol synthesis by squalene synthase inhibitors does not induce myotoxicity in vitro. Toxicol. Appl. Pharmacol. 145:91-98.

13. Matzno, S., et al. 1997. Inhibition of cholesterol biosynthesis by squalene epoxidase inhibitor avoids apoptotic cell death in L6 myoblasts. J. Lipid Res. 38:1639-1648.

14. Kaufmann, P., et al. 2006. Toxicity of statins on rat skeletal muscle mitochondria. Cell. Mol. Life Sci. 63:2415-2425.

15. Nambudiri, A.M., Ranganathan, S., and Rudney, H. 1980. The role of 3-hydroxy-3-methylglutaryl coenzyme A reductase activity in the regulation of ubiquinone synthesis in human fibroblasts. J. Biol. Chem. 255:5894-5899.

16. Berthold, H.K., et al. 2006. Effect of ezetimibe and/ or simvastatin on coenzyme Q10 levels in plasma: a randomised trial. Drug Saf. 29:703-712.

17. Lamperti, C., et al. 2005. Muscle coenzyme Q10 level in statin-related myopathy. Arch. Neurol. 62:1709-1712.

18. Mitch, W.E., and Goldberg, A.L. 1996. Mechanisms of muscle wasting. The role of the ubiquitin-proteasome pathway. N. Engl. J. Med. 335:1897-1905.

19. Attaix, D., et al. 2005. Altered responses in skeletal 
muscle protein turnover during aging in anabolic and catabolic periods. Int. J. Biochem. Cell Biol. 37:1962-1973.

20. Lecker, S.H., et al. 2004. Multiple types of skeletal muscle atrophy involve a common program of changes in gene expression. FASEB J. 18:39-51.

21. Sacheck, J.M., et al. 2007. Rapid disuse and denervation atrophy involve transcriptional changes similar to those of muscle wasting during systemic diseases. FASEB J. 21:140-155.

22. Gomes, M.D., Lecker, S.H.,Jagoe, R.T., Navon, A., and Goldberg,A.L. 2001. Atrogin-1, a muscle-specificF-box protein highly expressed during muscle atrophy. Proc. Natl. Acad. Sci. U. S. A. 98:14440-14445.

23. Bodine, S.C., et al. 2001. Identification of ubiquitin ligases required for skeletal muscle atrophy. Science. 294:1704-1708.

24. Adams, V., et al. 2007. Myocardial expression of Murf- 1 and MAFbx after induction of chronic heart failure: Effect on myocardial contractility. Cardiovasc. Res. 73:120-129.

25. Li, H.H., et al. 2004. Atrogin-1/muscle atrophy F-box inhibits calcineurin-dependent cardiac hypertrophy by participating in an SCF ubiquitin ligase complex. J. Clin. Invest. 114:1058-1071. doi:10.1172/JCI200422220.

26. Bdolah, Y., Segal, A., Tanksale, P., Karumanchi, S.A., and Lecker, S.H. 2007. Atrophy-related ubiquitin ligases atrogin-1 and MuRF-1 are associated with uterine smooth muscle involution in the postpartum period. Am. J. Physiol. Regul. Integr. Comp. Physiol. 292:R971-R976.

27. Frolov, A., et al. 2003. Response markers and the molecular mechanisms of action of Gleevec in gastrointestinal stromal tumors. Mol. Cancer Ther. 2:699-709.

28. Li, J.B., and Goldberg, A.L. 1976. Effects of food deprivation on protein synthesis and degradation in rat skeletal muscles. Am. J. Physiol. 231:441-448.

29. Tiao, G., Lieberman, M., Fischer, J.E., and Hasselgren, P.O. 1997. Intracellular regulation of protein degradation during sepsis is different in fast-and slow-twitch muscle. Am. J. Physiol. 272:R849-R856.

30. Baracos, V.E., DeVivo, C., Hoyle, D.H., and Goldberg, A.L. 1995. Activation of the ATP-ubiquitin-proteasome pathway in skeletal muscle of cachectic rats bearing a hepatoma. Am. J. Physiol. 268:E996-E1006.

31. Acharyya, S., et al. 2004. Cancer cachexia is regulated by selective targeting of skeletal muscle gene products. J. Clin. Invest. 114:370-378. doi:10.1172/ JCI200420174.

32. Spangenburg, E.E., and Booth, F.W. 2003. Molecular regulation of individual skeletal muscle fibre types. Acta Physiol. Scand. 178:413-424.

33. Peter, J.B., Barnard, R.J., Edgerton, V.R., Gillespie, C.A., and Stempel, K.E. 1972. Metabolic profiles of three fiber types of skeletal muscle in guinea pigs and rabbits. Biochemistry. 11:2627-2633.

34. Puigserver, P., et al. 1998. A cold-inducible coactivator of nuclear receptors linked to adaptive thermogenesis. Cell. 92:829-839.

35. Lin, J., Handschin, C., and Spiegelman, B.M. 2005. Metabolic control through the PGC-1 family of transcription coactivators. Cell Metab. 1:361-370.

36. Handschin, C., Rhee, J., Lin, J., Tarr, P.T., and Spiegelman, B.M. 2003. An autoregulatory loop controls peroxisome proliferator-activated receptor gamma coactivator 1alpha expression in muscle. Proc. Natl. Acad. Sci. U. S. A. 100:7111-7116.

37. Czubryt, M.P., McAnally, J., Fishman, G.I., and Olson, E.N. 2003. Regulation of peroxisome proliferator-activated receptor gamma coactivator 1 alpha (PGC-1 alpha) and mitochondrial function by MEF2 and HDAC5. Proc. Natl. Acad. Sci. U. S. A. 100:1711-1716.

38. Taylor, E.B., et al. 2005. Endurance training increases skeletal muscle LKB1 and PGC-1alpha protein abundance: effects of time and intensity. Am. J. Physiol. Endocrinol. Metab. 289:E960-E968.

39. Baar, K., et al. 2002. Adaptations of skeletal muscle to exercise: rapid increase in the transcriptional coactivator PGC-1. FASEB J. 16:1879-1886.

40. Russell, A.P., et al. 2003. Endurance training in humans leads to fiber type-specific increases in levels of peroxisome proliferator-activated receptor-gamma coactivator- 1 and peroxisome proliferator-activated receptor-alpha in skeletal muscle. Diabetes. 52:2874-2881.

41. Lin, J., et al. 2002. Transcriptional co-activator PGC-1 alpha drives the formation of slow-twitch muscle fibres. Nature. 418:797-801.

42. Sandri, M., et al. 2006. PGC-1alpha protects skeletal muscle from atrophy by suppressing FoxO3 action and atrophy-specific gene transcription. Proc. Natl. Acad. Sci. U. S. A. 103:16260-16265.

43. Pan, H.Y., et al. 1990. Comparative pharmacokinetics and pharmacodynamics of pravastatin and lovastatin. J. Clin. Pharmacol. 30:1128-1135.

44. Holstein, S.A., Knapp, H.R., Clamon, G.H., Murry, D.J., and Hohl, R.J. 2006. Pharmacodynamic effects of high dose lovastatin in subjects with advanced malignancies. Cancer Chemother. Pharmacol. 57:155-164.

45. Sandri, M., et al. 2004. Foxo transcription factors induce the atrophy-related ubiquitin ligase atrogin-1 and cause skeletal muscle atrophy. Cell. 117:399-412.

46. Sacheck, J.M., Ohtsuka, A., McLary, S.C., and Goldberg, A.L. 2004. IGF-1 stimulates muscle growth by suppressing protein breakdown and expression of atrophy-related ubiquitin-ligases, atrogin-1 and MuRF1. Am. J. Physiol. Endocrinol. Metab. 287:E591-E601.

47. Evenson, A.R., Fareed, M.U., Menconi, M.J., Mitchell, J.C., and Hasselgren, P.O. 2005. GSK-3beta inhibitors reduce protein degradation in muscles from septic rats and in dexamethasone-treated myotubes. Int. J. Biochem. Cell Biol. 37:2226-2238.

48. Birely, J., et al. 2005. Genetic screens for genes controlling motor nerve-muscle development and interactions. Dev. Biol. 280:162-176.

49. Poot, M., et al. 1996. Analysis of mitochondrial morphology and function with novel fixable fluorescent stains. J. Histochem. Cytochem. 44:1363-1372.

50. Siddals, K.W., Marshman, E., Westwood, M., and Gibson, J.M. 2004. Abrogation of insulinlike growth factor-I (IGF-I) and insulin action by mevalonic acid depletion: synergy between protein prenylation and receptor glycosylation pathways. J. Biol. Chem. 279:38353-38359.

51. Chen, Y.W., et al. 2005. Early onset of inflammation and later involvement of TGFbeta in Duchenne muscular dystrophy. Neurology. 65:826-834.

52. Phillips, P.S., Phillips, C.T., Sullivan, M.J., Naviaux, R.K., and Haas, R.H. 2004. Statin myotoxicity is associated with changes in the cardiopulmonary function. Atherosclerosis. 177:183-188.

53. Seachrist, J.L., Loi, C.M., Evans, M.G., Criswell, K.A., and Rothwell, C.E. 2005. Roles of exercise and pharmacokinetics in cerivastatin-induced skeletal muscle toxicity. Toxicol. Sci. 88:551-561.

54. Schaefer, W.H., et al. 2004. Evaluation of ubiquinone concentration and mitochondrial function relative to cerivastatin-induced skeletal myopathy in rats. Toxicol. Appl. Pharmacol. 194:10-23.

55. Tintignac, L.A., et al. 2005. Degradation of MyoD mediated by the SCF (MAFbx) ubiquitin ligase. J. Biol. Chem. 280:2847-2856.

56. Serrano, A.L., et al. 2001. Calcineurin controls nerve activity-dependent specification of slow skeletal muscle fibers but not muscle growth. Proc. Natl. Acad. Sci. U. S. A. 98:13108-13113.

57. Gadbut, A.P., Caruso, A.P., and Galper, J.B. 1995. Differential sensitivity of C2-C12 striated muscle cells to lovastatin and pravastatin. J. Mol. Cell. Cardiol. 27:2397-2402.

58. Bruckert, E., Hayem, G., Dejager, S., Yau, C., and Begaud, B. 2005. Mild to moderate muscular symptoms with high-dosage statin therapy in hyperlipidemic patients--the PRIMO study. Cardiovasc. Drugs Ther. 19:403-414.

59. Bruckert, E., Verpilleux, M.P., Dejager, S., and Isaacsohn, J. 2005. Comparison of efficacy and safety assessment of fluvastatin in patients $<65$ years versus $>$ or $=65$ years of age. Am. J. Cardiol. 96:1142-1148.

60. Fisher, J.E., et al. 1999. Alendronate mechanism of action: geranylgeraniol, an intermediate in the mevalonate pathway, prevents inhibition of osteoclast formation, bone resorption, and kinase activation in vitro. Proc. Natl. Acad. Sci. U. S. A. 96:133-138.

61. Wysowski, D.K., and Chang, J.T. 2005. Alendronate and risedronate: reports of severe bone, joint, and muscle pain. Arch. Intern. Med. 165:346-347.

62. Mootha, V.K., et al. 2004. Erralpha and Gabpa/b specify PGC-1alpha-dependent oxidative phosphorylation gene expression that is altered in diabetic muscle. Proc. Natl. Acad. Sci. U. S. A. 101:6570-6575.

63. Patti, M.E., et al. 2003. Coordinated reduction of genes of oxidative metabolism in humans with insulin resistance and diabetes: Potential role of PGC1 and NRF1. Proc. Natl. Acad. Sci. U. S. A. 100:8466-8471.

64. Waclawik, A.J., Lindal, S., and Engel, A.G. 1993. Experimental lovastatin myopathy. J. Neuropathol. Exp. Neurol. 52:542-549.

65. Westwood, F.R., Bigley, A., Randall, K., Marsden, A.M., and Scott, R.C. 2005. Statin-induced muscle necrosis in the rat: distribution, development, and fibre selectivity. Toxicol. Pathol. 33:246-257.

66. Thompson, P.D., et al. 1997. Effect of prolonged exercise training without weight loss on high-density lipoprotein metabolism in overweight men. Metabolism. 46:217-223.

67. Unnikrishnan, D., and Satish, B. 2005. Exertioninduced rhabdomyolysis in a patient on statin therapy. Nephrol. Dial. Transplant. 20:244.

68. Sinzinger, H., Wolfram, R., and Peskar, B.A. 2002. Muscular side effects of statins. J. Cardiovasc. Pharmacol. 40:163-171.

69. Flint, O.P., Masters, B.A., Gregg, R.E., and Durham, S.K. 1997. HMG CoA reductase inhibitor-induced myotoxicity: pravastatin and lovastatin inhibit the geranylgeranylation of low-molecular-weight proteins in neonatal rat muscle cell culture. Toxicol. Appl. Pharmacol. 145:99-110.

70. Okuno, Y., et al. 2002. Distal elements are critical for human CD34 expression in vivo. Blood. 100:4420-4426.

71. Yang, H., Menconi, M.J., Wei, W., Petkova, V., and Hasselgren, P.O. 2005. Dexamethasone upregulates the expression of the nuclear cofactor p300 and its interaction with $\mathrm{C} / \mathrm{EBPb}$ ta in cultured myotubes. J. Cell. Biochem. 94:1058-1067.

72. Hanai, J., et al. 2002. Endostatin is a potential inhibitor of Wnt signaling. J. Cell Biol. 158:529-539.

73. Westerfield, M. 1993. The zebrafish book: a guide for the laboratory use of zebrafish Danio (Brachydanio) rerio. University of Oregon Press. Eugene, Oregon, USA. 363 pp.

74. Kimmel, C.B., Ballard, W.W., Kimmel, S.R., Ullmann, B., and Schilling, T.F. 1995. Stages of embryonic development of the zebrafish. Dev. Dyn. 203:253-310.

75. Crow, M.T., and Stockdale, F.E. 1986. The developmental program of fast myosin heavy chain expression in avian skeletal muscles. Dev. Biol. 118:333-342.

76. Devoto, S.H., Melancon, E., Eisen, J.S., and Westerfield, M. 1996. Identification of separate slow and fast muscle precursor cells in vivo, prior to somite formation. Development. 122:3371-3380. 